\title{
DUODENAL ULGERS IN THE NEW BORN
}

BY

W. S. CRAIG, B.Sc., M.D., M.R.C.P.Ev.

(From the Department of Child Life and Health, University of Edinburgh.)

Paterson ${ }^{1}$ and Hurst and Stewart" have drawn attention to the small number of cases of duodenal ulcer in infants, and particularly in new-born infants, reported in the literature of this country. The following two examples are recorded, not merely on account of the rarity of the condition, but also because there are points of particular interest in each case.

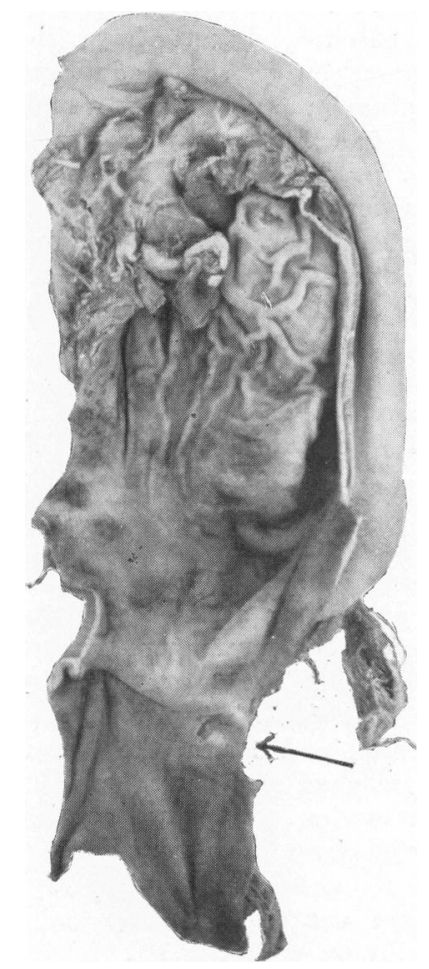

Fíg. 1.-Duodenal ulcer in the new born.

Baby S. delivered by Caesarean section.

Death $4 \frac{1}{2}$ days after delivery. The arrow indicates the position of the ulcer.

\section{Case reports.}

Gase 1.-Baby S. (male). Born in the Royal Maternity Hospital, Edinburgh, May 8th, 1933. After an uneventful pregnancy the child was delivered at term by Caesarean section on account of contracted pelvis. Caesarean section had also been carried out at the birth of the mother's one previous child. The child weighed $7 \mathrm{lb}$. $11 \mathrm{oz}$. at birth and was asphyxiated but responded to treatment. Sickness occurred after each feed during the first three days of life, and pallor was noted. 
On May 11th there was no sickness, but vomiting began again the next day and the stools were relaxed and offensive for the first time. The abdomen became distended and the firm flexion of the thighs and nature of the cry suggested that the child was in pain. Vomiting persisted, and on the evening of May 13th the child collapsed and became semi-comatose. There was no improvement during the night and death occurred early on the morning of May 14th. No blood was seen at any time in the vomitus or stools, and temperature was normal throughout.

At the autopsy the body was that of a full-time infant. Abdomen was prominent; the abdominal cavity contained a large amount of viscid, pale yellow fluid and a number of curd-like objects suggestive of stomach contents. Early peritonitis was present. A thin fibrinous exudate covered spleen and upper surface of liver, and fibrinous adhesions bound the proximal duodenum to the adjacent gallbladder and pancreas. There was no evidence of perforation in either stomach or intestines. A small ulcer $2 \mathrm{~mm}$. in diameter was present in the posterior wall of the duodenum (Fig. 1) immediately distal to the pylorus and extending deeply to the muscle coat. The margins were slightly reddened and sharply defined, giving the ulcer a punched-out appearance. The pyloric sphincter was normal in appearance. Chemical tests revealed the presence of occult blood in a specimen of the stools. An extensive haemorrhage had occurred into the right suprarenal with complete destruction of the medullary substance. A less severe haemorrhage had also taken place in the left suprarenal. Umbilicus and umbilical veins were healthy. Petechial haemorrhages were present in the visceral layer of the pleurae and pericardium. In the brain a haemorrhage of limited extent, not associated with thrombosis of vessels, was found in relation to the supero-lateral aspect of the right occipital lobe. The extravasation had occurred mainly into the subarachnoid space. There was no growth on culture of the heart blood.

Microscopically there was local destruction of the mucosa, submucosa and superficial layers of the muscle coat of the stomach at the site of the ulcer, associated with some mono-nuclear cell infiltration of the mucous lining at the margins. There was no haemorrhage or thrombosis.

Case 2.-Baby D. (male) born in the Elsie Inglis Memorial Hospital, Edinburgh, at 8 a.m., April 17th, 1933 . The mother was a primipara, aged 21 years; her husband was not the father of the child. Pregnancy was uneventful. Labour lasted twelve and three-quarter hours, light chloroform anaesthesia being administered from the beginning of the second stage. Instruments were not used. The ohild was twelve weeks premature according to the mother's dates, but was calculated to be only four to six weeks premature by Dr. Elliott who was in charge of the case. Weight at birth was $5 \mathrm{lb}$. $10 \mathrm{oz}$. Healthy in appearance, the child was put to the breast for the first time at 5 p.m. on the day of delivery. Further breast feeds were given at 5 a.m. and 9 a.m. the following day, and shortly after the second of these a meconium stool was passed which was slightly blood stained. One hour later (10.15 a.m.) a motion was passed consisting almost solely of blood. Ten c.c. of maternal blood was given intra-muscularly. On two further occasions blood was passed rectally and the child sank rapidly, dying at 6 p.m. on April 8th, 34 hours after delivery. There was no vomiting and the child took the breast vigorously until within eight hours of death.

At the autopsy the body was that of a small premature infant. Cranial sutures were widely separated and the bones in the region of the parietal and occipital eminences were thin and parchment-like. A large blood clot filled the distended duodenum and a considerable amount of unclotted blood was present throughout the rest of the small intestine and colon. At a point on the lower surface of the duodenum $5 \mathrm{~mm}$. distal to the pylorus there was a small ulcer $3 \mathrm{~mm}$. in diameter, with swollen, overhanging, ragged margins (Fig. 2). The floor of the ulcer consisted of the submucous layer and attached to a small vessel in it were a few strands of clotted blood. A second ulcer was present, situated in the posterior wall of the duodenum. It presented a similar appearance to the other but was smaller, less sharply defined and no vessels were visible in its base. There was no hypertrophy of the pyloric sphincter. Heart blood was sterile on culture. 


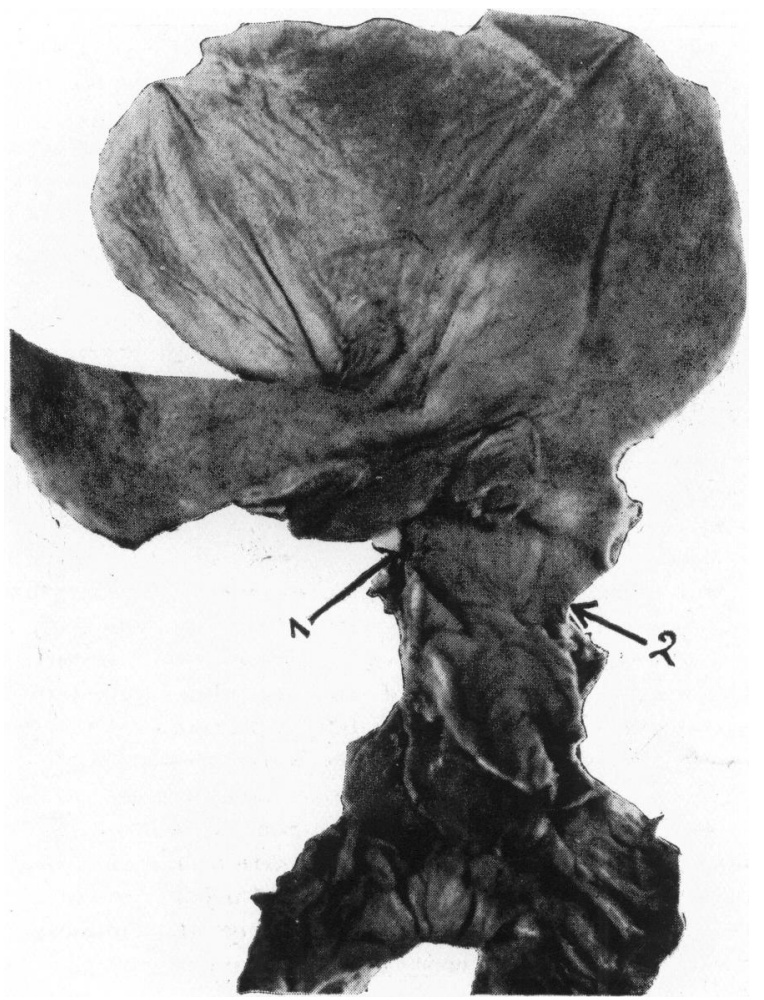

Fig. 2.-Duodenal ulcer in the new born. Baby D. Six weeks premature. Death 36 hours after birth. The arrows indicate the position of the two ulcers.

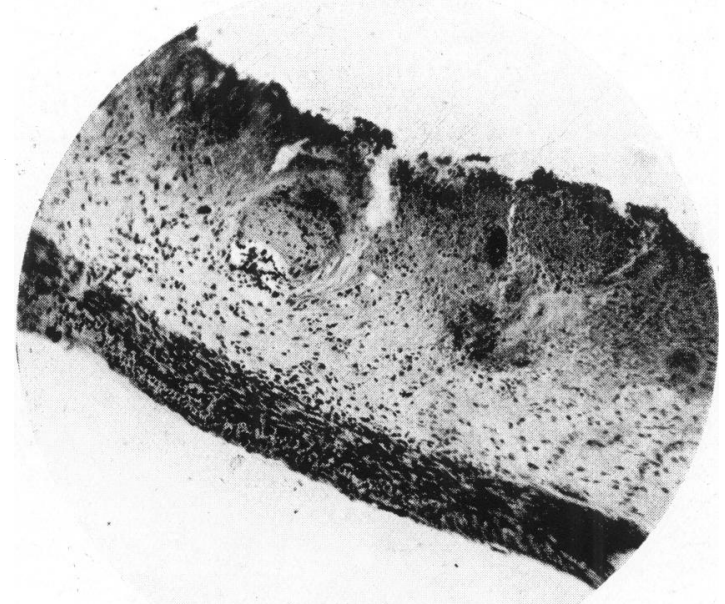

Fig. 3.-Duodenal ulcer in the new born. Baby D. Low power microphotograph $(x, 80)$ of larger ulcer showing numerous thrombi in vessels of the submucous layers. 


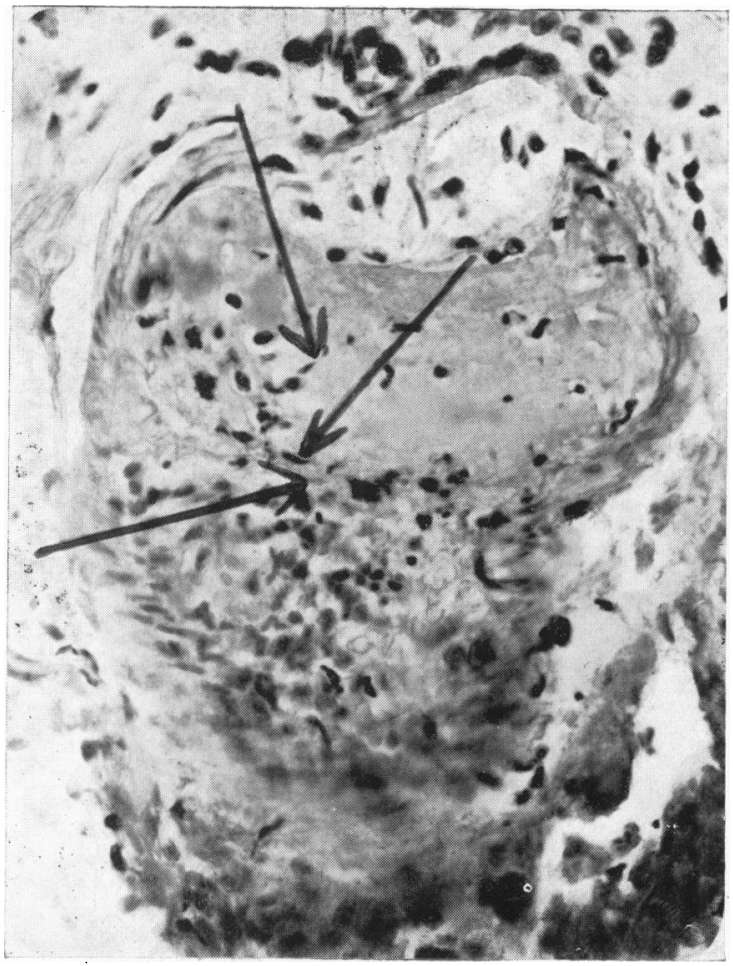

Fig. 4.-Duodenal ulcer in the new born. Baby D. High power microphotograph $(\times 440)$ of larger ulcer showing large thrombus undergoing commencing organization. Fibroblasts are indicated by arrows.

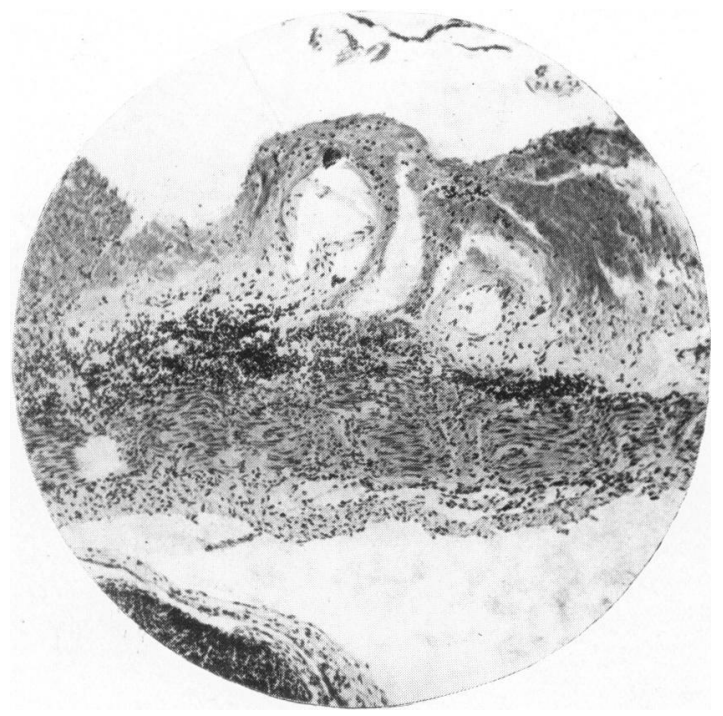

Fig. 5.-Duodenal ulcer in the new born. Low power microphotograph $(x \quad 80)$ of smaller ulcer, showing haemorrhage into the submucous layer, 
Microscopically there was (Fig. 3, 4, and 5) generalized thrombosis of the vessels in the submucous tissues deep to the ulcers. Fibroblasts could be seen in several of the thrombi, pointing to commencing organization. Destruction of the mucosa was complete in the region of the ulcer and there was early necrosis in the submucosa. In the smaller ulcer there was, in addition, moderately extensive haemorrhage into the submucous layer. There was no evidence of an inflammatory reaction,

\section{Discussion.}

The two cases, both new-born infants, provide a striking clinical contrast. In the second, melaena was the only clinical evidence of the condition apart from the eventual collapse; there was no vomiting, and death occurred rapidly. In the first there was no visible haemorrhage, but vomiting was a persistent feature from birth, occult blood was present in the stools and there was increasing pallor.

An equally striking contrast is presented by the pathological findings in the two cases. In the first case, in addition to an ulcer in the duodenum, haemorrhage of a gross nature had taken place into the suprarenals and further evidences of a tendency to bleeding were present in the serous surfaces of the thorax and in the brain. No lesion of the alimentary tract other than the ulcer in the duodenum and no possible source of swallowed blood were found, and it can be assumed that the occult blood in the stool taken at autopsy owed its origin to the ulcer.

The association of duodenal ulceration with suprarenal haemorrhage suggests the existence of some common aetiological factor. The possibility of suprarenal haemorrhage being the result of sepsis has been mentioned by Hamill $^{3}$, Holt', Gunson", and Goldzieher ${ }^{6}$. Reuss ${ }^{7}$ considered that fever in the mother is a contributory factor, while Magnus" is of the opinion that eclampsia in the mother plays the same rôle. External violence and trauma are mentioned as causes by Langmead ${ }^{9}$, Goldzieher ${ }^{6}$ and others.

In the case under consideration a culture of the heart blood was sterile and no focus of infection was found, either during life or post mortem. The pregnancy was uneventful. Delivery was by Caesarean section so that the haemorrhage cannot be attributed to birth trauma. None of the usual hypotheses are available in explanation of the occurrence of suprarenal haemorrhage in the first case (Baby S.). Asphyxia was a feature of the child's condition at birth and this has been given as a cause of haemorrhage in the new born by Pearce ${ }^{10}$ and Arnold ${ }^{1}$. As these writers suggest, it is possible that the increased pressure and venous congestion associated with the asphyxia give rise to the haemorrhages. Apart from the primary factor causing bleeding, the spontaneous nature of the haemorrhages, their multiplicity and severity point to the case being one of haemorrhagic disease of the new born, with the duodenal ulcer as one of the bleeding points. The history of vomiting from birth suggests that the ulcer was present either at or immediately after birth, and the suprarenal haemorrhage probably occurred at the time of collapse on the evening prior to death. In the absence of any other explanation it seems probable that the peritonitis followed perforation of the ulcer which subsequently became walled off. 
In the second case (Baby D.) the peptic ulcers in the duodenum were the only pathological finding at autopsy, indicating that the haemorrhage leading to death was the result of a purely local condition. Microscopically the ulcers in this case differed from that in Baby S. in that thrombosis of the subjacent vessels was present. The thrombi showed no inflammatory changes: culture of heart blood was sterile, and no septic focus was found post mortem. The occurrence of thrombosis in the under-lying capillaries in association with peptic ulcers in the new born has already been recorded by Schmidt $^{12}$, Theile ${ }^{13}$, Harrison ${ }^{14}$, and others. On the assumption that thrombosis of vessels in the vicinity is a primary factor in the ulceration, various suggestions as to its causation have been advanced which have been summarized by Theile $^{13}$. It has been argued that retrograde thrombosis follows low pressure in the venous circulation occurring with the circulatory changes at birth. Such an hypothesis fails to explain why thrombi are not also found in other sites such as the large sinuses usually involved in marantic thrombosis.

Landau $^{15}$ considers that the thrombosis forming at the site of compression of the umbilical vein gives rise to emboli which become lodged in the region of the ulcer. Emboli originating from such a source might be expected to involve other organs, more especially the liver and lungs. At autopsy on Baby D. no embolic infarcts were found in other viscera. Infarcts are not commonly met with in post mortems on the new born and, in the absence of any anatomical explanation for emboli showing a selective affinity for the gastric or duodenal submucosa, Landau's hypothesis fails to explain the presence of thrombosis in association with peptic ulcers.

Thrombosis of the vessels deep to a peptic ulcer has also been attributed to the absorption of toxins by the gastro-duodenal mucous membrane. In the case of Baby D. the mother's condition was healthy and her pregnancy ran a normal course, and it is unlikely that any abnormal absorption of toxins by the foetal circulation occurred in utero. Delivery was spontaneous. Clinical and post-mortem examinations of the child excluded the possibility of either local or general infection. The child's only nourishment was obtained from the mother's breasts which were perfectly healthy. Nothing in the history of either the mother or the child suggested a likelihood of toxic absorption.

Finkelstein ${ }^{16}$ has suggested that thrombosis arises as the result of the action of the stomach juices on the vessel walls. As Theile ${ }^{13}$ points out, the acidity of the suckling's stomach is relatively slight; in a new-born premature infant gastric secretion must be limited and it is unlikely that irritation by the stomach juices was a primary factor giving rise to the thrombosis in the case of Baby $D$.

The wasted condition of infants in whom peptic ulcers have been found has been often noted. In large numbers the co-existence of organic disease has accounted for the extreme debility. Syphilis ${ }^{13}$, tuberculosis ${ }^{1,2,13}$, nephritis ${ }^{2,13}$, eczema ${ }^{17}$, and burns ${ }^{18}$ have all been found in association with wasting and a peptic ulcer. Schmidt ${ }^{12}$ records cases of peptic ulcer in 10 
children suffering from various forms of severe sepsis which had undermined their general condition. Schmidt ${ }^{12}$, Theile ${ }^{13}$, Helmholz ${ }^{19}$, Selinger ${ }^{20}$ and Paterson $^{1}$ are all of the opinion that the lowered resistance following organic disease is an important predisposing factor in connection with the occurrence of peptic ulcers.

The relatively large number of recorded cases of peptic ulcers occurring in marasmic infants is important in this connection. The association has been noted by Theile ${ }^{13}$, Veeder ${ }^{21}$, Selinger ${ }^{20}$, Paterson ${ }^{1}$ and Schmidt ${ }^{12}$, and is referred to by Hurst and Stewart ${ }^{2}$. Holt ${ }^{4}$ emphasized the correspondence between the age of maximum incidence of peptic ulcer in children and the age of highest death rate from marasmus, while Moynihan ${ }^{22}$ is of the opinion that peptic ulcers are common in atrophic infants. In the absence of organic disease the cause of marasmus is to be looked for in difficulties associated with the feeding. The error lies in incorrect feeding or in a congenital constitutional weakness on the part of the infant. The essential factor is that feeding has been unsuited to the digestive power of the particular infant.

Baby D's birth was premature. In the normal course of events the development of the organs of digestion would have proceeded for a further four to six weeks. Nine hours after birth and at regular intervals afterwards, at a time when normally a placental circulation should have provided means of nourishment, the child was put to the breast. Complete emptying of the breasts of colostrum was favoured by the ready way in which the child fixed and by the vigour of his sucking. In this way the child was required to digest and assimilate a fluid characteristically rich in protein and unsuited to its imperfectly developed power of digestion. A state of affairs was present, therefore, similar to that already described in connection with certain marasmic infants. With Baby D., however, the basic constitutional weakness was not of a problematical nature but existed definitely in virtue of its premature birth. The case is of importance in that it demonstrates that prematurity may be associated with the same factors predisposing towards duodenal or gastric ulceration as are met with in marasmic infants.

The two cases here recorded indicate that ulceration of the duodenum in the new born may arise in one of two ways. The local destruction of tissue involved in the ulceration may be an end result of a haemorrhagic tendency on the part of the infant. The ulcer is a manifestation of a general condition. It is probable that extravasation of blood into the deeper tissues leads to necrosis and digestion of the superficial mucous membrane. On the other hand, as in the case of Baby D., the ulcer may be an entirely local lesion, the result of an insult to the duodenal mucous membrane, the insult arising from the ingestion of food beyond the assimilative powers of the infant. In all probability local reaction takes the form of vascular spasm and this would explain the occurrence of thrombosis. Muir ${ }^{23}$, dealing with peptic ulcers in adults, says that spasmodic contraction of small vessels is the only possible explanation of associated vascular lesions. The natural outcome of the thrombosis is the necrosis and ulceration of the tissues related to the impaired circulation. 
My thanks are due to Professor Charles McNeil for giving permission to record the first case and to Dr. Margaret Martin for supplying the clinical details in connection with the second case. I also wish to acknowledge the assistance given me by Dr. A. R. Macgregor in preparing this paper.

\section{REFERENCES.}

1. Paterson, D., Lancet, Lond., 1922, i, 63.

2. Hurst, A. F., \& Stewart, M. J., Gastric \& Duodenal Ulcer, Oxford, 1929.

3. Hammil, S. M., Arch. Pediat., New York, 1901, XVIII, 81.

4. Holt, L. E., \& Howland, J., Diseases of Infancy \& Childhood, 9th Ed., 1926.

5. Gunson, E. B., Proc. Roy. Soc. Med., Lond., 1914-15, VIII, 54.

6. Goldzieher, M. A., \& Greenwald, H. M., Am. J. Dis. Child., Chicago, 1928, XXXVI, 324.

7. v. Reuss, A. R., The Diseases of the New Born, quoted by Arnold, D.P., Am. J. Dis. Child., Chicago, 1930, XL, 1053.

8. Magnus, G., Klin. Wchnschr., Berlin, 1911, LVIII, 1119.

9. Langmead, F. S., Diseases of Children, Garrod, A. E., et alii, 2nd Ed., Lond., 1929.

10. Pearce, M. O., Abt's Paediatrics, Philadelph., II, 353.

11. Arnold, D. P., Am. J. Dis. Child., Chicago, 1930, XL, 1053.

12. Schmidt, W., Klin. Wchnschr., Berlin, 1913, L, 593.

13. Theile, P., Ergebn. d. inn. Med. u. Kinderh., Berlin, 1919, XVI, 302.

14. Harrison, L. P., Arch. Dis. Childh., Lond., 1931, VI, 245.

15. Landau, H., Uber Melaena der Neugeborenen, Breslau, 1874, quoted by Theile, P., Ergebn. d. inn. Med. u. Kinderh., Berlin, 1919, XVI, 302.

16. Finkelstein, H., Klin. Wchnschr., Berlin, 1908, XLVI, 1950.

17. Borland, H. N., Lancet, Lond., 1903, ii, 1034.

18. Ashby, I. E., Med. J. Austral., Sydney, 1917, I, 183.

19. Helmholz, H. F., Arch. Pediat., New York, 1909, XXVI, 661: Deutsche med. Wchnschr., Leipzig, 1909, XXXV, 534.

20. Selinger, J., Ann. Surg., Philadelph., 1932, X(VI, 204.

21. Veeder, B. S., Am. J. Med. Sc., Philadelph., 1914, CXLVIII, 709.

22. Moynihan, B. G. A., Duodenal Ulcer, Philadelph., 2nd Ed., 1912.

23. Muir, R., Text Book of Pathology, Lond., 1926, 422. 